\title{
Influence of hyaluronic acid on bacterial and fungal species, including clinically relevant opportunistic pathogens
}

\author{
Andrea Ardizzoni - Rachele G. Neglia • Maria C. Baschieri • \\ Claudio Cermelli • Manuela Caratozzolo • Elena Righi • \\ Beniamino Palmieri · Elisabetta Blasi
}

Received: 13 May 2011/ Accepted: 28 July 2011/Published online: 4 September 2011

(C) Springer Science+Business Media, LLC 2011

\begin{abstract}
Hyaluronic acid (HA) has several clinical applications (aesthetic surgery, dermatology, orthopaedics and ophtalmology). Following recent evidence, suggesting antimicrobial and antiviral properties for HA, we investigated its effects on 15 ATCC strains, representative of clinically relevant bacterial and fungal species. The in vitro system employed allowed to assess optical density of broth cultures as a measure of microbial load in a time-dependent manner. The results showed that different microbial species and, sometimes, different strains belonging to the same species, are differently affected by HA. In particular, staphylococci, enterococci, Streptococcus mutans, two Escherichia coli strains, Pseudomonas aeruginosa, Candida glabrata and $C$. parapsilosis displayed a HA dosedependent growth inhibition; no HA effects were detected in E. coli ATCC 13768 and C. albicans; S. sanguinis was favoured by the highest HA dose. Therefore, the influence of HA on bacteria and fungi warrants further studies aimed at better establishing its relevance in clinical applications.
\end{abstract}

\footnotetext{
A. Ardizzoni · R. G. Neglia - M. C. Baschieri - C. Cermelli

M. Caratozzolo · E. Righi

Dipartimento di Scienze di Sanità Pubblica, Università

di Modena e Reggio Emilia, Modena, Italy

B. Palmieri

Dipartimento di Chirurgia Generale, Università di Modena e Reggio Emilia, Modena, Italy

E. Blasi

Dipartimento di Laboratori, Anatomia Patologica e Medicina

Legale, Università di Modena e Reggio Emilia, Modena, Italy

E. Blasi $(\bowtie)$

Istituti Biologici ( $2^{\circ}$ piano), Via Campi 287, 41125 Modena, Italy

e-mail: elisabetta.blasi@unimore.it
}

\section{Introduction}

Hyaluronic acid (HA) is a glycosaminoglycan made up of glucuronic acid and $\mathrm{N}$-acetylglucosamine disaccharide units. It is a uniform, linear, unbranched and tiled molecule, with highly variable length and molecular weight (up to $10^{6} \mathrm{Da}$ ). It is abundant in skin (up to 56\%) and in connective tissues, with a turnover ranging from several hours to a few days depending on tissues. Also, HA constitutes one of the main components of extracellular matrices. In some tissues, like vitreous humour and synovial fluid, HA is the primary responsible for the function and the physical characteristics associated with these substances. Its length, coupled to its high hydrating property, allows many HA polymers to organize in a reticular structure, which in turn produces a molecular framework. Such scaffolding, besides supporting tissues' tone and shape, acts as a filter to prevent the diffusion of high molecular weight substances and dissemination of infectious agents [1].

Because of its biological properties, including non immunogenicity which is an essential prerequisite for biocompatibility, HA and its derivatives are broadly used in pharmacy and medicine, especially in segment surgery as well as in wound and burn therapy [2]. In particular, HA plays a prominent role in wound-healing processes, as it is naturally angiogenic when degraded to small fragments, which act as "danger signals" [3-6]. Also, HA is known to promote early inflammation, which is critical for initiating wound healing, while at later times it contributes to matrix stabilization, thus participating to downregulation of inflammatory reactions $[5,6]$.

HA is mainly used in aesthetic surgery and in dermatology, where it is injected, together with collagen proteins, to eliminate wrinkles and to prevent skin aging. HA has 
many other therapeutical applications, including its use in ophtalmic surgery of the vitreous body, in otologic surgery as a regenerator after perforation of the tympanic membrane and, in orthopedics, as an antiphlogistic lubricant and a preserving agent of joint's synovial fluid [1].

HA is administered as a high molecular weight compound; such a formulation prevents degradation by physiological hyaluronidases, thus allowing long time persistence of HA and no production of small fragments. Notoriously, the latter would promote undesirable inflammatory reactions due to their direct interactions with the cells of the innate immune system. A better understanding of the wide spectrum role of HA, both in biological and pathological conditions, has been recently reviewed [7].

In spite of such a wide employment, only a few studies have been conducted to assess the effects, if any, of HA on infectious agents. Initial in vitro evidence ascribes to HA an antiviral activity with respect to HSV2 [8], rubella virus, Newcastle disease virus and vesicular stomatitis virus [9]. Recently, a study conducted by our group on the in vitro antiviral and virucidal activity of a high molecular weight HA demonstrates that, depending upon the virus considered, HA may exert strong (on Coxsackievirus B5, Mumps virus and Influenza Virus A/H1N1), mild (on HSV1 and Porcine parvovirus) or even no antiviral activity (on Adenovirus-5, HHV-6, Porcine Reproductive and Respiratory Syndrome Virus), whereas no virucidal activity was ever observed [10].

As for bacteria, scant and sometimes controversial data are available. In one hand, it has been reported that staphylococci and streptococci are able to grow in presence of Na-hyaluronate $(0.7 \%)$; accordingly, it has been suggested that, by producing hyaluronidases in the presence of HA, such bacteria may immediately obtain low molecular weight sugars, useful as nutrients [11]. In line with this hypothesis, a decrease in viscosity of extracellular matrix has been recorded, thus likely facilitating microbial spread; as a matter of fact, most hyaluronidase producing Gram positive bacteria are able to cause skin and/or mucosal infections. Furthermore, Zhang and coworkers [12] have shown that, in HA-enriched growth medium, Streptococcus pyogenes upregulates several virulence factors emphasizing that the streptococcal ability to degrade HA should be considered as a virulence factor itself. On the other hand, several papers provide evidence that HA can exert bacteriostatic effects on staphylococci and streptococci, depending on HA concentration and molecular weight [11, 13-15]. Additional studies claim that Pseudomonas aeruginosa is not affected by HA [14-16]. Finally, up to date, no direct bactericidal or fungicidal effects by HA have been observed [13, 17].

Here, by an in vitro assay, we investigated the effects of HA on a group of bacterial and fungal strains, mostly known to be clinically relevant as common cause of human infections, either at mucosal/skin level or in tissues as responsible of iatrogenic infections associated with clinical practice. We show that HA can have different effects on different microbial species and sometimes also on different strains belonging to the same species.

\section{Materials and methods}

\subsection{Culture media}

The following liquid and solid media were used for strain maintenance and viable count assessment: Brain Heart Infusion (BHI) and Brain Heart Agar (BHA) (Oxoid, Hampshire, U.K.) for the bacterial strains, Sabouraud Dextrose Broth (SDB-Biokar Diagnostics, France) and Sabouraud Dextrose Agar (SDA-Oxoid, Hampshire, U.K.) for the yeast strains. The following selective media were used to identify the bacterial strains: Mannitol Salt Agar (MSA-Fluka Biochemika, Germany) for staphylococci; Mitis salivarius Agar (MsA-Becton-Dickinson, MD, USA) for oral streptococci; McConkeyAgar (Oxoid, Hampshire, U.K.) for Escherichia coli and Pseudomonas aeruginosa; Bile Aesculin Agar (BAA-Oxoid, Hampshire, U.K.) for enterococci.

\subsection{Hyaluronic acid}

High molecular weight $(1837 \mathrm{kDa})$ hyaluronic acid in powder (batch \#A04A 13/03/2008, Shiseido Co. Ltd., Kakegawa Factory, Japan) was gently provided by IBSA (Institute Biochemique SA, Lugano, $\mathrm{CH}$ ). HA was diluted in sterile saline solution to obtain a final concentration of $8 \mathrm{mg} / \mathrm{ml}$. After a complete resuspension, (obtained through a $24 \mathrm{~h}$ stirring) and sterilization by using $0,45 \mu \mathrm{m}$ filters (Schleicher \& Schuell, Germany), HA solution was aliquoted and frozen at $-20^{\circ} \mathrm{C}$ until use.

\subsection{Microorganisms}

The following 11 bacterial and 4 fungal strains were employed: Enterococcus faecalis (ATCC 29212), E. hirae (ATCC 10541), Escherichia coli (ATCC 10536, 13762 and 25922), Pseudomonas aeruginosa (ATCC 15442 and 27853), Staphylococcus aureus (ATCC 25923), S. epidermidis (ATCC 12228), Streptococcus mutans (ATCC 25175), S. sanguinis (ATCC 10556), Candida albicans (ATCC 90028 and 90029), C. glabrata (ATCC 90030) and C. parapsilosis (ATCC 22019).

In order to perform experiments, microbial cells (from stocks kept at $-80^{\circ} \mathrm{C}$ ) were revitalized in BHI or SDB and then checked for purity on selective agar plates. From 
isolated colonies, subcultures were expanded in broth, for $18 \mathrm{~h}$ (bacteria) and $24 \mathrm{~h}$ (yeasts), up to a microbial concentrations of $1.5 \times 10^{8} \mathrm{CFU} / \mathrm{ml}$ for the bacteria and $1.5 \times 10^{6} \mathrm{CFU} / \mathrm{ml}$ for the fungi, according to the optical density (O.D.) of the 0.5 McFarland standard [18]. Finally, the microbial suspensions were prepared in BHI (bacterial strains) or SDB (fungal strains), in order to obtain the working concentrations of $5 \times 10^{6} \mathrm{CFU} / \mathrm{ml}$ or $5 \times 10^{3} \mathrm{CFU} / \mathrm{ml}$ for the bacteria and $5 \times 10^{5} \mathrm{CFU} / \mathrm{ml}$ for the fungi. As an internal control, viable counting was performed.

McFarland standards were prepared, as detailed elsewhere [18], and used as reference for densitometric assays, using the Tecan Sunrise microplate reader (Tecan, Austria).

\subsection{Evaluation of HA effect on microorganisms}

The assay was performed in 96-well microplates (Corning Inc., NY, USA); $100 \mu \mathrm{l} /$ well of microbial suspensions were added to $100 \mu \mathrm{l} /$ well of saline (control) or HA, at the final concentrations of $4 \mathrm{mg} / \mathrm{ml}$ (HA1), $2 \mathrm{mg} / \mathrm{ml}$ (HA2), $1 \mathrm{mg} / \mathrm{ml}$ (HA3), $0.5 \mathrm{mg} / \mathrm{ml}$ (HA4) and $0.25 \mathrm{mg} / \mathrm{ml}$ (HA5). The blank wells contained only BHI, with saline solution, or HA at the above mentioned concentrations. McFarland standards were dispensed in each plate $(200 \mu \mathrm{l} / \mathrm{well})$, as internal controls. Every condition was tested in quadruplicate wells. The plates were incubated at $37^{\circ} \mathrm{C}$ in a moist chamber. The O.D. values were read immediately (time 0) and at hourly intervals (with the exception of night hours) for at least $72 \mathrm{~h}$. At the end of each experiment, viable counts were assessed by Colony Forming Units (CFUs) assay in some wells, randomly sampled.

O.D. values were measured at two different wavelengths (595 $\mathrm{nm}$ for the bacteria and $540 \mathrm{~nm}$ for the yeasts) by means of a microplate reader (Tecan, Austria).

\subsection{Data analysis}

Data obtained from the O.D. readings were used to draw charts where O.D. was expressed as a function of time. Each point of the curves represented the average value of 4 replicates (subtracted of the blank) of the same experimental condition. The standard deviation values were not reported to facilitate charts' reading. Statistical analyses were performed at approximately $6 \mathrm{~h}$ intervals: at each fixed time, analysis of variance (ANOVA) and Bonferroni post hoc test were carried out to assess overall difference in O.D. readings obtained from different groups in relation to control groups. Statistical significance was set at $P<0.05$, while $P$ values $<0.01$ were indicative of highly statistically significant differences.

\section{Results}

\subsection{Effects of HA on staphylococcal strains}

In order to evaluate the effect of HA on staphylococcal strains, S. aureus ATCC 25923 (Fig. 1a, b) and S. epidermidis ATCC 12228 (Fig. 1c, d) were employed at two starting bacterial loads, $5 \times 10^{3} \mathrm{CFU} / \mathrm{ml}$ (Fig. 1a, c) and $5 \times 10^{6} \mathrm{CFU} / \mathrm{ml}$ (Fig. 1b, d). At time 0 , bacteria were exposed to increasing concentrations of HA (from $0.25 \mathrm{mg} / \mathrm{ml}$ to $4 \mathrm{mg} / \mathrm{ml}$, as detailed in Sect. 2) and then assessed for O.D. at different times, up to $72 \mathrm{~h}$. As shown in Fig. 1, each staphylococcal species displayed a peculiar trend, in the kinetics of O.D.; the curve profiles appeared very similar (panel A vs. panel B; panel C vs. panel D) and only slightly shifted towards later times, when considering the lower bacterial starting concentration. In particular, $S$. aureus O.D. increased by the time in all groups; yet, when compared to controls, HA-treated bacteria showed delayed and lower O.D. curves depending on the HA doses (panels A and B). When assessing S. epidermidis in the presence of HA (panels $\mathrm{C}$ and $\mathrm{D}$ ), we found little or no O.D. increase in bacteria exposed to the three highest HA doses, while at $0.5 \mathrm{mg} / \mathrm{ml}$ (HA4), a gradual increment in O.D. occurred after $30 \mathrm{~h}$ and reached the maximal levels, comparable to the controls' values, between 50 and $60 \mathrm{~h}$. In both experimental conditions (panels $\mathrm{C}$ and $\mathrm{D}$ ), control group's O.D. partially decreased at 70-72 h.

Statistical analysis, performed on data depicted in panels $\mathrm{B}$ and D, showed that the differences in O.D., between $S$. aureus exposed to each HA concentration and the control group, were highly significant at all the time points; the only exception was the lowest dose (HA5), where significance was lost after $6 \mathrm{~h}$ of incubation (Fig. 1, bottom left panel). As for S. epidermidis (Fig. 1, bottom right panel), the differences in O.D. between HA-treated and control groups were statistically significant at all the concentrations with the exception of HA5, where significance was lost after $10 \mathrm{~h}$ of incubation and it was regained at $72 \mathrm{~h}$ $(P<0.05)$. It should be noted that, at $70-72 \mathrm{~h}$, the significance occurring between control and HA4 or HA5 O.D. was actually due to control levels being significantly below those observed in HA-treated groups.

In parallel groups, viable cells were determined as CFUs (viable CFUs) in HA-treated and untreated groups and then compared to the bacterial concentrations estimated by O.D. (O.D. CFUs). As an example, for S. epidermidis at $72 \mathrm{~h}$, the O.D. CFUs versus viable CFUs were $5.3 \times 10^{9}$ versus $2.4 \times 10^{9}$ (controls), $6.8 \times 10^{9}$ versus $3.3 \times 10^{9}$ (HA5treated bacteria), $6.4 \times 10^{9}$ versus $1.2 \times 10^{9}$ (HA4-treated bacteria), $2.7 \times 10^{9}$ versys $1.2 \times 10^{9}$ (HA3-treated bacteria), $1.8 \times 10^{9}$ versus $0.6 \times 10^{9}$ (HA2-treated bacteria), $1.4 \times 10^{9}$ versus $0.1 \times 10^{9}$ (HA1-treated bacteria). Based 

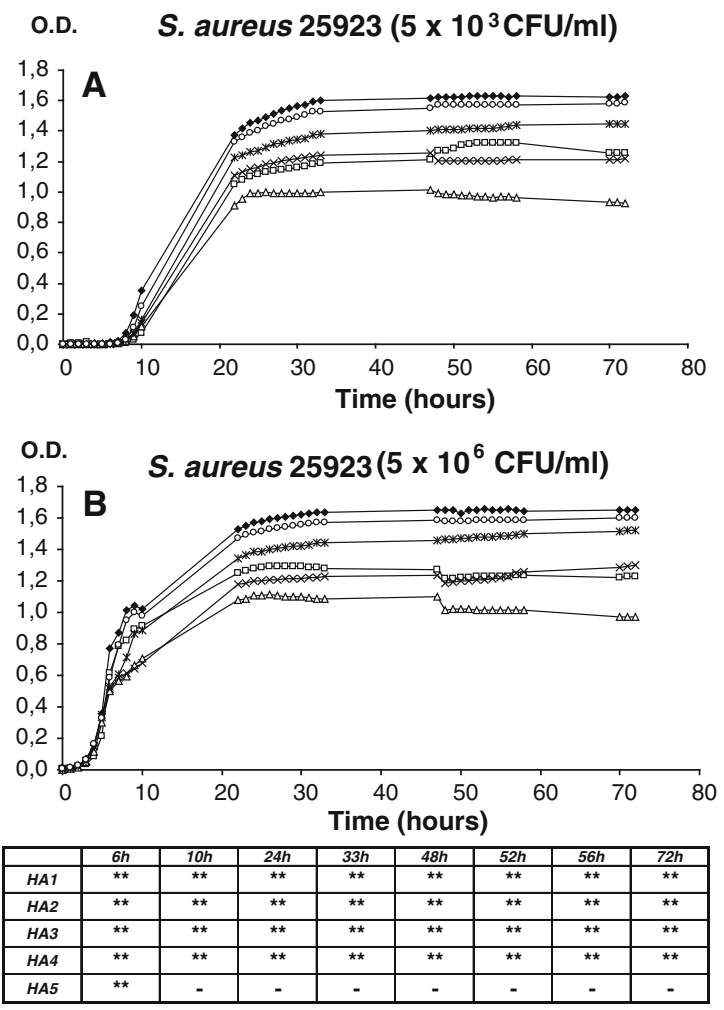

Fig. 1 Effects of HA on staphylococcal strains. Kinetic curves of O.D. in S. aureus ATCC 25923 (panels a and b) and S. epidermidis ATCC 12228 (panels c and d). The bacteria were exposed to five different HA concentrations $(4,2,1,0.5$ and $0.25 \mathrm{mg} / \mathrm{ml}$ ) or to medium (control). The bacteria were employed at two different starting concentrations, $5 \times 10^{3} \mathrm{CFU} / \mathrm{ml}$ (panels a and c) and $5 \times 10^{6} \mathrm{CFU} / \mathrm{ml}$ (panels $\mathbf{b}$ and d). Lower panels: statistical significance between HA-treated and untreated $S$. aureus and

on the similarities between viable CFUs and O.D. CFUs, hereafter, the O.D. reading was taken as measure of bacterial load, while viable CFUs were occasionally evaluated.

\subsection{Effects of HA on streptococcal and enterococcal strains}

In order to evaluate the effects of HA on streptococci and enterococci, 4 strains were selected: S. mutans ATCC 25175, S. sanguinis ATCC 10556, E. faecalis ATCC 29212 and E. hirae ATCC 10541. Figure 2 depicts the O.D. values of HA-treated and untreated groups as a function of time.

HA displayed a strong inhibitory effect on $S$. mutans, at all the doses from $24 \mathrm{~h}$ onwards (Fig. 2a). In particular, while control group slowly and gradually increased, reaching the plateau level after $48 \mathrm{~h}$, the HA-treated groups showed a delayed O.D. increase irrespectively of the doses, reaching plateau levels much below the control group $(P<0.01)$, at all the time-points.

As shown in Fig. 2b, also $S$. sanguinis was influenced by HA, but to a different extent. In particular, between 6 and
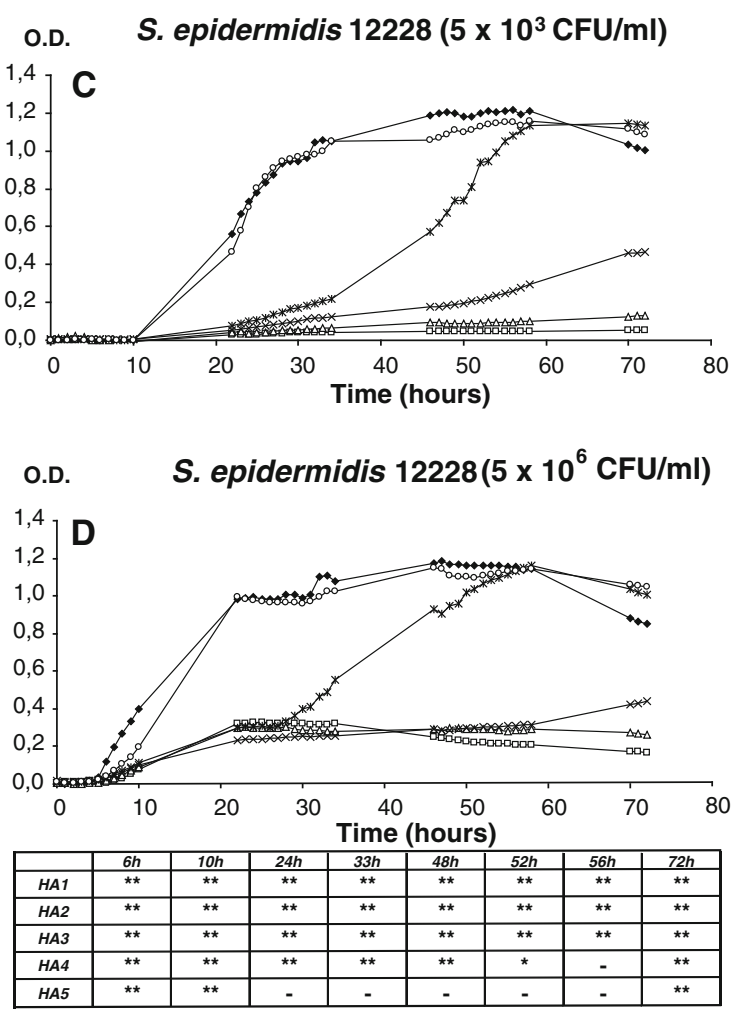

S. epidermidis, at the starting concentration of $5 \times 10^{6} \mathrm{CFU} / \mathrm{ml}$. Eight different time points are shown. filled diamond controls (no hyaluronic acid); square HA1 (4 mg/ml hyaluronic acid); triangle HA2 $(2 \mathrm{mg} / \mathrm{ml}$ hyaluronic acid); times HA3 $(1 \mathrm{mg} / \mathrm{ml}$ hyaluronic acid); snowflake HA4 $(0.5 \mathrm{mg} / \mathrm{ml}$ hyaluronic acid); circle HA5 $(0.25 \mathrm{mg} / \mathrm{ml}$ hyaluronic acid). **Highly significant $(P<0.01)$; *significant $(P<0.05)$; - not significant $(P>0.05)$

$10 \mathrm{~h}$, a significant dose-dependent reduction of O.D. was observed (groups HA1, HA2, HA3 vs control group). Unexpectedly, a drastic and transient (20-35 h) increment in O.D. was detected in HA1-treated streptococci. Later on, as well as for all the other groups, no statistically significant differences were recorded.

As depicted in Fig. 2c, E. faecalis showed a rapid and sharp increase in O.D. reaching the maximal values at $10 \mathrm{~h}$ (control and HA5), $22 \mathrm{~h}$ (HA4), or $26 \mathrm{~h}$ (HA3), followed by a slight time-related decrease in all the three treated groups as well as in the control. Differently, O.D. of HA2-treated bacteria after an initial increase $(0-10 \mathrm{~h})$ remained at the same O.D. levels up to $36 \mathrm{~h}$; then, a gradual increment occurred and maximal O.D. levels were achieved at the latest time-points (from $70 \mathrm{~h}$ on). In contrast, E. faecalis exposed at the highest dose (HA1) showed O.D. levels that, after an initial peak (reaching at $10 \mathrm{~h}$ values about half of controls) gradually decreased by the time. Statistically significant differences were consistently recorded.

As shown in Fig. 2d, HA displayed a clear inhibitory dose-dependent effect against $E$. hirae, being the O.D. of 

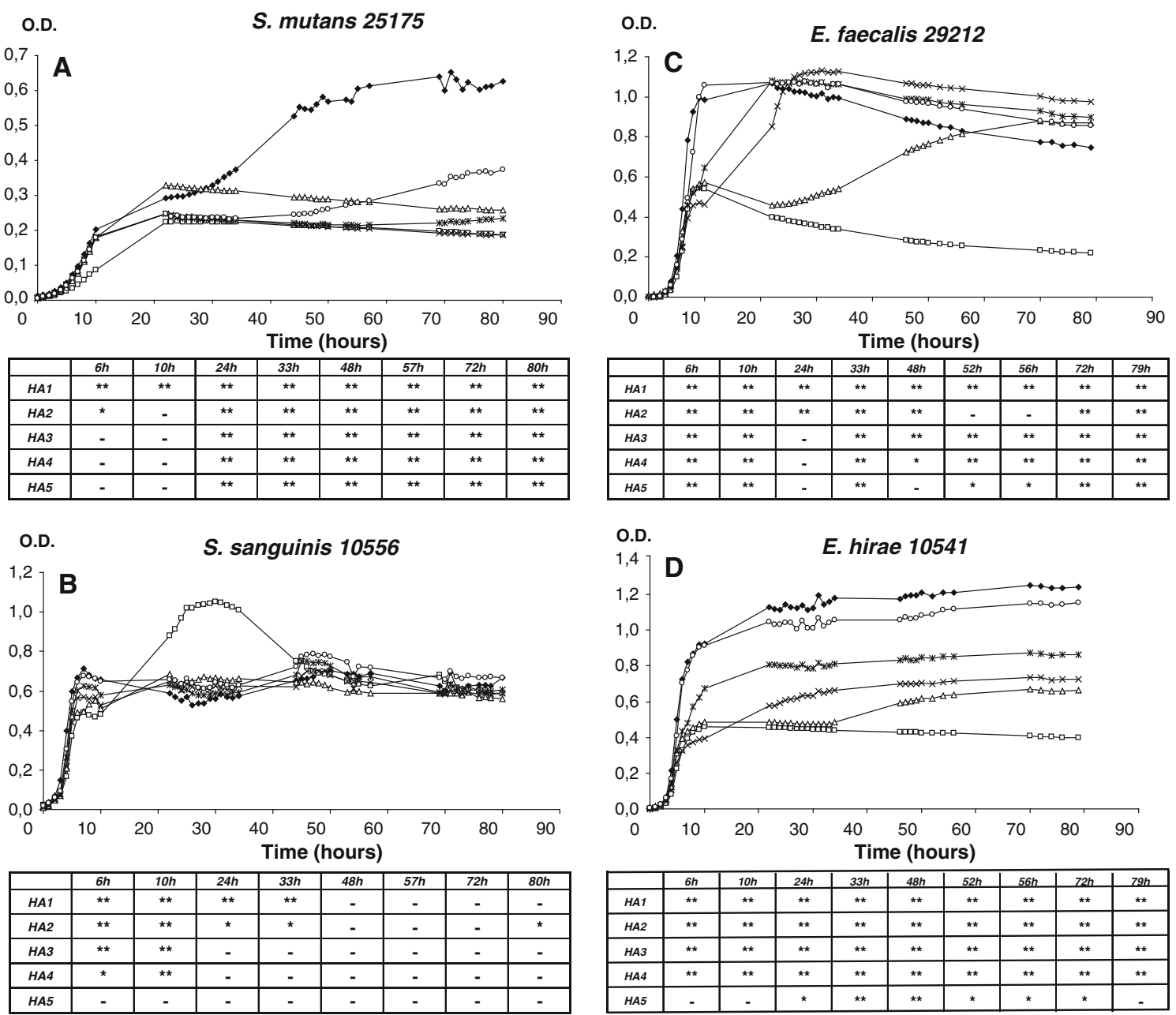

Fig. 2 Effects of HA on streptococcal and enterococcal strains. Kinetic curves of O.D. in S. mutans ATCC 25175 (panel a), S. sanguinis ATCC 10556 (panel b), E. fecalis ATCC 29212 (panel c) and E. hirae ATCC 10541 (panel d). The bacteria $\left(5 \times 10^{6} \mathrm{CFU} / \mathrm{ml}\right)$ were exposed to five different HA concentrations $(4,2,1,0.5$ and $0.25 \mathrm{mg} / \mathrm{ml})$ or to medium (control). Statistical significance between HA-treated and untreated

controls always significantly above that of treated bacteria; in particular, such effect was dose-dependent and significant at all the times and with all the HA doses (with the exception of a partial effect displayed by HA5 group, at most of the times).

\subsection{Effects of HA on Gram negative bacteria}

The effect of HA on Gram negative bacteria was evaluated on three E. coli strains (ATCC 10536, 13768 and 25922) and two P. aeruginosa strains (ATCC 15442 and 27853).

As depicted in Fig. 3a, HA had dose-dependent inhibitory effects on E. coli 10536 O.D. levels. In most of the cases, differences between controls and HA-treated bacteria were highly significant. A dose-dependent inhibition

bacteria was evaluated for each strain at the indicated times. filled diamond controls (no hyaluronic acid); square HA1 (4 mg/ml hyaluronic acid); triangle $\mathrm{HA} 2$ ( $2 \mathrm{mg} / \mathrm{ml}$ hyaluronic acid); times $\mathrm{HA} 3$ $(1 \mathrm{mg} / \mathrm{ml}$ hyaluronic acid); snowflake HA4 $(0.5 \mathrm{mg} / \mathrm{ml}$ hyaluronic acid); circle HA5 $(0.25 \mathrm{mg} / \mathrm{ml}$ hyaluronic acid). **Highly significant $(P<0.01)$; *significant $(P<0.05)$; - not significant $(P>0.05)$

was also observed for E. coli 25922 (Fig. 3b), but only at the highest HA concentrations (HA1, HA2 and HA3). These 3 groups always exhibited highly significant differences from controls at all times, while HA4- and HA5treated groups either displayed significance at some time points or they were not significant. HA had little or no effect towards E. coli 13768 (Fig. 3c). In this case, the difference between controls and treated cells was significant only for HA1 and HA2 groups.

Finally, as for $P$. aeruginosa ATCC 15442, a high (around 2.0) O.D. increment occurred in control groups (Fig. 3d); HA treatment did not affect the kinetic profiles although, at the highest doses, significant differences were observed in stationary phase levels with respect to controls. Similar results were obtained with the strain ATCC 27853 (data not shown). 

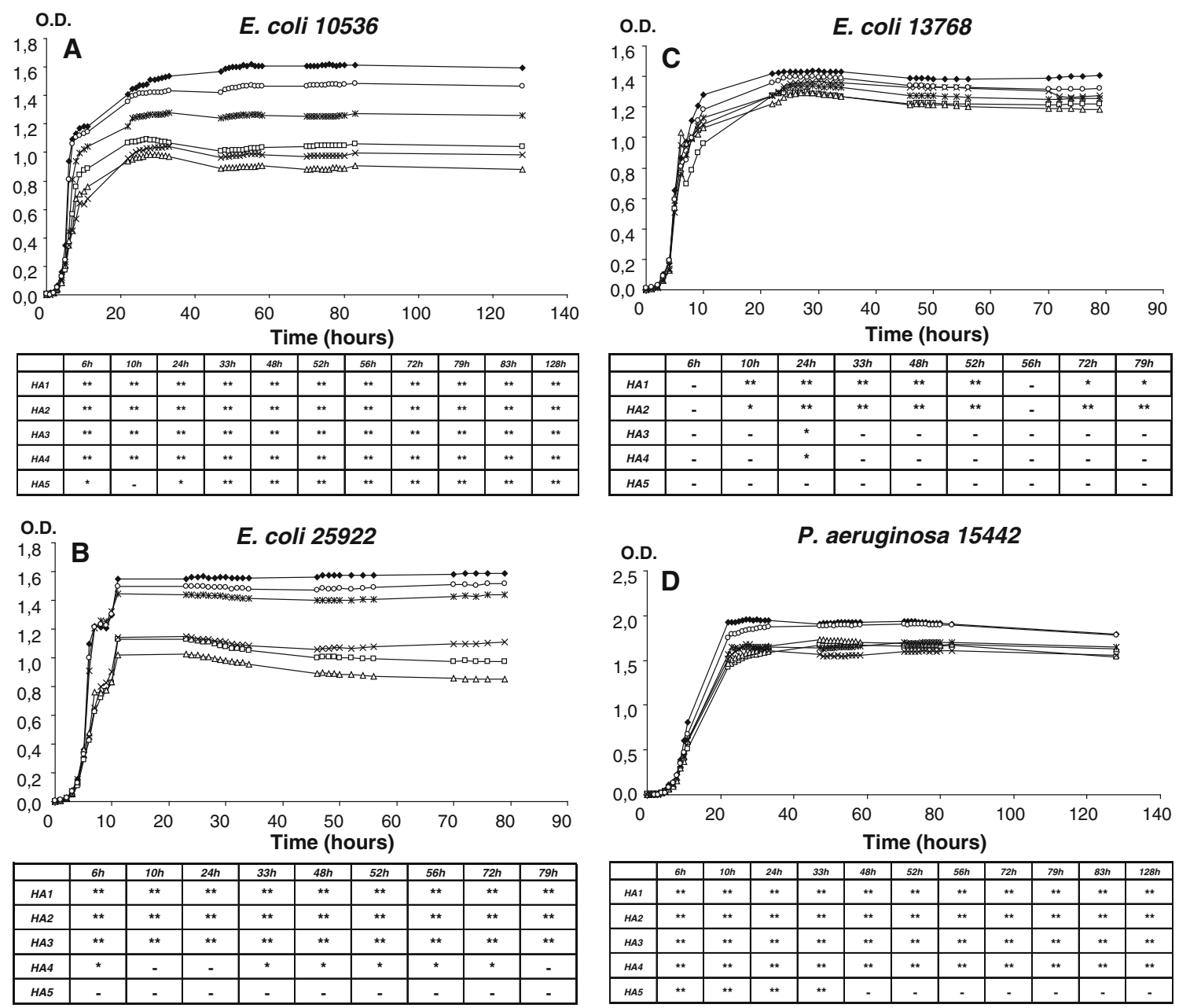

Fig. 3 Effects of HA on Gram negative bacteria. Kinetic curves of O.D. in E. coli ATCC 10536 (panel a), E. coli ATCC 25922 (panel b), E. coli ATCC 13768 (panel c) and P. aeruginosa ATCC 15442 (panel d). The bacteria $\left(5 \times 10^{6} \mathrm{CFU} / \mathrm{ml}\right)$ were exposed to five different $\mathrm{HA}$ concentrations $(4,2,1,0.5$ and $0.25 \mathrm{mg} / \mathrm{ml})$ or to medium (control). Statistical significance between HA-treated and untreated bacteria

\subsection{Effects of HA on fungal cells}

Four Candida spp. strains were assessed for their sensitivity to HA: C. albicans ATCC 90028 and 90029, C. glabrata ATCC 90030, and C. parapsilosis ATCC 22019. In particular, HA caused negligible O.D. variations amongst treated groups and controls, on the $C$. albicans ATCC 90029 (Fig. 4a). Accordingly, no statistically significant differences could be highlighted between controls and HA-treated cells, the only exception being the fungi incubated with the highest HA concentration (HA1) up to $61 \mathrm{~h}$. A similar trend was obtained with $C$. albicans 90028 (data not shown).

Differently, as shown in Fig. 4b, C. glabrata was affected by $\mathrm{HA}$ in the range between 0.25 and $2 \mathrm{mg} / \mathrm{ml}$ (HA5 to HA2). Unexpectedly, yeast cells incubated with

was evaluated for each strain at the indicated times. filled diamond controls (no hyaluronic acid); square HA1 (4 mg/ml hyaluronic acid); triangle HA2 (2 mg/ml hyaluronic acid); times HA3 $(1 \mathrm{mg} / \mathrm{ml}$ hyaluronic acid); snowflake HA4 (0.5 mg/ml hyaluronic acid); circle HA5 $(0.25 \mathrm{mg} / \mathrm{ml}$ hyaluronic acid $)$. **Highly significant $(P<0.01)$; *significant $(P<0.05)$; - not significant $(P>0.05)$

$4 \mathrm{mg} / \mathrm{ml}$ (HA1) showed O.D. values higher than the other HA-treated groups and just below control's O.D.. As a consequence most of the differences between HA-treated groups and controls were significant at all times, with the exception of HA1. As for HA-treated C. parapsilosis, O.D. values were consistently lower than controls' (Fig. 4c); the phenomenon was dose-dependent and highly significant for all the HA doses, but HA5.

\section{Discussion}

As a major component of the extracellular matrix in connective tissues, HA plays several physiological roles, such as structure maintenance, moisturizing, tissue lubrication and wound healing. These features, associated with an 
O.D. C. albicans 90029

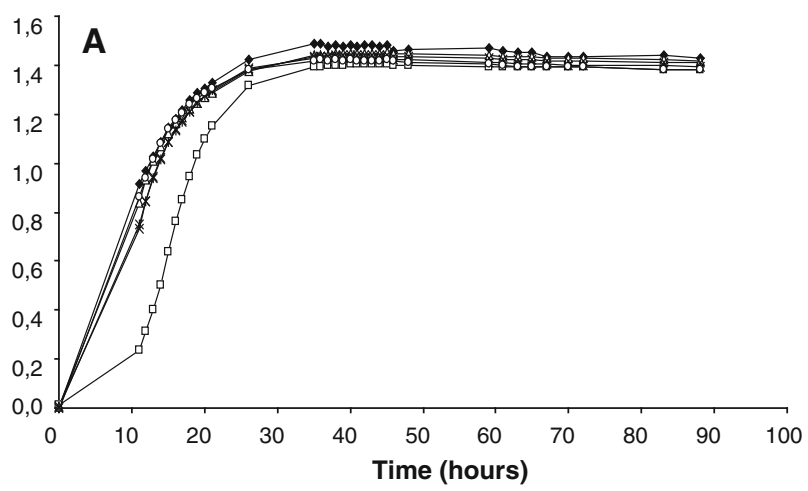

\begin{tabular}{|c|c|c|c|c|c|c|c|}
\hline & $12 \mathrm{~h}$ & $26 \mathrm{~h}$ & $36 \mathrm{~h}$ & $48 \mathrm{~h}$ & $61 \mathrm{~h}$ & $72 \mathrm{~h}$ & $88 \mathrm{~h}$ \\
\hline HA1 & $\star \star$ & - & $*$ & $\star$ & $\star \star$ & - & - \\
\hline HA2 & - & - & - & - & - & - & - \\
\hline HA3 & $\star$ & - & - & - & - & - & - \\
\hline HA4 & $\star$ & - & - & - & - & - & - \\
\hline HA5 & - & - & - & - & $*$ & - & - \\
\hline
\end{tabular}
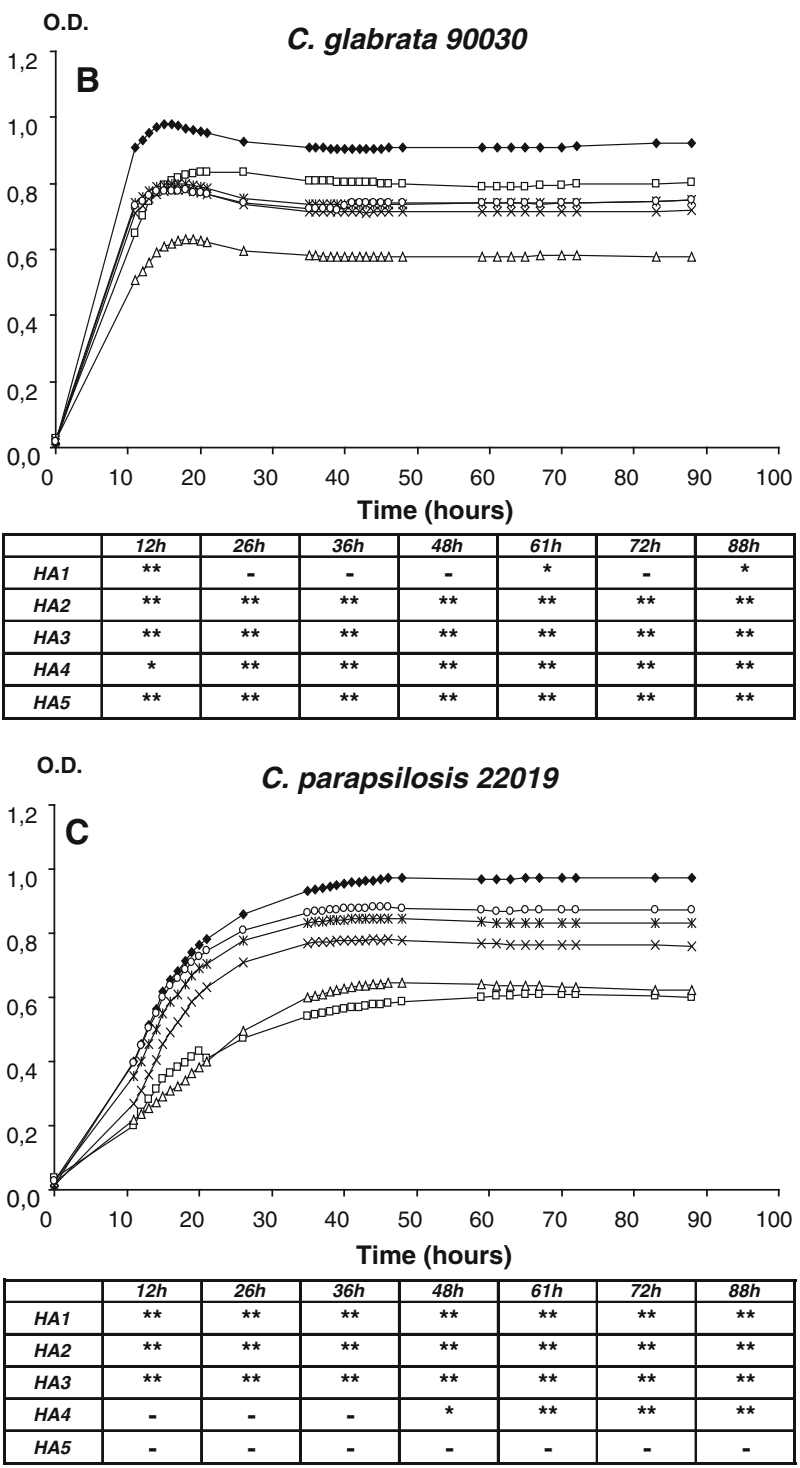

Fig. 4 Effects of HA on fungal cells. Kinetic curves of O.D. in C. albicans ATCC 90029 (panel a), C. glabrata ATCC 90030 (panel b) and $C$. parapsilosis ATCC 22019 (panel c). The fungi $\left(5 \times 10^{5}\right.$ $\mathrm{CFU} / \mathrm{ml})$ were exposed to five different HA concentrations $(4,2,1$, 0.5 and $0.25 \mathrm{mg} / \mathrm{ml}$ ) or to medium (control). Statistical significance between HA-treated and untreated fungi was evaluated for each strain at the indicated times. filled diamond controls (no hyaluronic acid); square HA1 (4 mg/ml hyaluronic acid); triangle HA2 $(2 \mathrm{mg} / \mathrm{ml}$ hyaluronic acid); times HA3 (1 mg/ml hyaluronic acid); snowflake HA4 $(0.5 \mathrm{mg} / \mathrm{ml}$ hyaluronic acid); circle HA5 $(0.25 \mathrm{mg} / \mathrm{ml}$ hyaluronic acid). **Highly significant $(P<0.01)$; *significant $(P<0.05)$; - not significant $(P>0.05)$

excellent safety profile, prompted the use of hexogenous HA in medical practice as well as in aesthetic and cosmetic fields [1]. Recently, experimental evidence on antiviral and antimicrobial properties of HA $[8-10,13,15,19]$ has added further appeal to HA-containing products used in clinical practice.

Here, we show that a high molecular weight HA has different effects on different bacterial and yeast cells. In particular, we employed a widely accepted in vitro system $[13,16,17]$ that allows simultaneous evaluation of several HA doses, in multiple replicates that are all repeatedly assessed for at least $72 \mathrm{~h}$; by this approach real long-term kinetic curves, devoid of inter-assay variations, are obtained.

By preliminary experiments, we demonstrate that the in vitro O.D. variations in bacterial cultures exposed to HA do not depend on the initial bacterial density; as a matter of fact, the overall trends are very similar in $S$. epidermidis and $S$. aureus when starting with $5 \times 10^{3}$ or $5 \times 10^{6}$ $\mathrm{CFU} / \mathrm{ml}$ cell concentration; only an expected delay in curve slopes is recorded, but interestingly the final plateau levels are comparable between groups treated with the same HA dose, irrespectively of the initial bacterial load. Moreover, the superimposable results obtained in terms of viable CFUs strengthen the relevance of the O.D. as a parameter to investigate the HA effects on microbial growth. By this model, we have assessed several bacterial and fungal species, for at least $72 \mathrm{~h}$ and in the presence of different HA doses, thus, providing a wide-range in vitro picture of what HA may represent in terms of microbial promotion/ inhibition.

Specifically, our data indicate that $S$. aureus growth is affected by HA, with an inhibition proportional to the HA concentration employed; the only exception is the HA1 dose which returns intermediate inhibitory effects. This somehow unexpected result consistently occurs also irrespectively of the initial microbial load; whether the highest HA concentration may induce in S. aureus yet unravelled metabolic pathways capable of reverting the initial inhibitory effects remains an open question. In any case, these results add insights to the controversial literature reporting 
different effects of HA on staphylococci, ranging from growth promotion [11], to no changes $[14,16]$ to a clear inhibitory effect [15]. Whether such differences are to be ascribed to the different $S$. aureus strains used in each experimental model remains an open question.

As for $S$. epidermidis, our results show that HA exerts clear dose-dependent inhibition, highlighted by the fact that O.D.s of bacteria treated with the highest doses of HA remain at very low levels. Thus, in accordance with previous works [14, 15], we show that HA exerts an inhibitory effect on S. epidermidis. Interestingly, both in previous [15] and present study, the same strain (ATCC 12228) has been employed; also, the HA concentration tested $(4 \mathrm{mg} / \mathrm{ml})$ by Carlson et al. [15] corresponds to the highest concentration used in our experimental model (HA1). Moreover, our study provides novel information in terms of dosedependency: in addition to the inhibitory effect observed at the highest dose, HA causes a remarkable dose-dependent delay in bacterial growth, especially in the presence of HA4 (till $30 \mathrm{~h}$ ) or HA3 (till $70 \mathrm{~h}$ ). Whether the long-lasting low O.D. levels, observed in HA1- and HA2-treated groups and interpreded as growth inhibition, may possibly start to recover and increase again at time points over $72 \mathrm{~h}$ remains to be established.

Both $S$. mutans and $S$. sanguinis are known to produce hyaluronidases [11], thus potentially taking advantage by the presence of HA. Unexpectedly, here, we show that the O.D.s of HA-treated S. mutans remain significantly below the O.D. of the untreated counterparts, while only the HA5treated group shows a slow and gradual increase after $48 \mathrm{~h}$. As above mentioned for $S$. epidermidis, we cannot exclude that also $S$. mutans exposed to the high HA doses may restart to grow again later on. Interestingly, $S$. sanguinis exhibits a very peculiar response to the highest HA dose; indeed, while most of the curves of the treated groups are indistinguishable from controls, at all the time-points tested, HA1-treated group shows a quick and transient overraise in O.D. between 20 and $35 \mathrm{~h}$; this profile may be explained by assuming that HA1 allows $S$. sanguinis an optimal growth, followed by a drastic bacterial death possibly related to consumption of nutrients and/or accumulation of toxic catabolites, as suggested by the sharp decrease in O.D.. Furthermore, the initial lag time, observed between 8 and $12 \mathrm{~h}$ in HA1-treated S. sanguinis, suggests that upon HA stimulation, an inducible hyaluronidase activity is produced, thus providing at later times a growth advantage responsible for the O.D. peak observed at $20-35 \mathrm{~h}$.

As for enterococci and HA, no literature data are available yet. Our results indicate that HA has different effects, depending on the species. In detail, we show that the effect of HA on E. faecalis is consistent with a delay in growth rather than with a long-lasting inhibition. Unlike
HA5-treated bacteria, which exhibit O.D. values similar to controls, bacteria exposed to the higher concentrations of HA show dose-related and time-dependent delays in O.D. curve raise that in all the cases reach values similar to control. A peculiar profile occurs in HA1-treated group that, after an initial $(0-10 \mathrm{~h})$ increase, remains at O.D. levels much below those observed for any other group up to $80 \mathrm{~h}$; these findings imply that a major inhibitory effect occurs; yet, according to the profiles shown with the other HA doses, we may not exclude a novel growth at times over $80 \mathrm{~h}$.

By our model, E. hirae appears to be inhibited in a dosedependent manner, with the HA-treated bacteria showing O.D. levels significantly below controls, at all the timepoints tested. Thus, we provide the first evidence that this species is susceptible to HA inhibitory effects. It is worth noting that, although rarely isolated in clinical setting, E. hirae, closely related to E. faecalis, is a reference species in European Standard Procedures to assess efficacy of disinfectants [19-21].

A previous paper claims that $E$. coli is unable to use Healon (an ophtalmologic medicament containing 0.5\% $\mathrm{HA}$ ) as a nutrient, since no growth has been recorded at 24 , 48 and $72 \mathrm{~h} \mathrm{[11].} \mathrm{Our} \mathrm{findings} \mathrm{expand} \mathrm{and} \mathrm{partially} \mathrm{con-}$ tradict such information, showing that HA effects on E. coli are strain-dependent. In our hands, two (10536 and 25922) out of the three $E$. coli strains assessed are strongly inhibited by increasing doses of HA; differently, E. coli 13768 is poorly susceptible to HA and only at the highest doses (HA1 and HA2), where some significance has been recorded. Therefore, we may conclude that HA effects on E. coli should be carefully investigated and established according to the strain considered.

It is generally acknowledged that $P$. aeruginosa is not affected by HA [14, 16, 19]. This species does not produce hyaluronidases [22], but it synthesizes its own HA, which indeed is one of the main components of Pseudomonasinduced biofilm [23]. In contrast, Carlson et al. [15] describe a growth inhibition in $P$. aeruginosa exposed to HA for $6 \mathrm{~h}$. Here we show that, with the exception of the lowest dose (HA5), the O.D. curves of all the HA-treated groups reach plateau values consistently lower than controls. However, although statistical analysis reveals significance between groups, the O.D.s of both treated and untreated bacteria have limited variations, ranging between 1.5 and 2.0. From here, we can conclude that HA per se does not have relevant antibacterial effects on $P$. aeruginosa, although some disturbance by HA at concentrations $\geq 0.5 \mathrm{mg} / \mathrm{ml}$ is detectable by our experimental system.

Tang et al. [16] showed that $C$. albicans growth is not affected by sodium hyaluronate, while Kang et al. [17] have shown fungistatic but not fungicidal effects in a $20 \mathrm{~h}$ experimental model. By investigating multiple doses and 
measuring microbial O.D. till $90 \mathrm{~h}$ in two different strains, our present findings explain the apparent dichotomy between the results provided by the literature. A significant delay in C. albicans O.D. increase is detected initially and only with the highest HA dose, but no effects are evident at later times, irrespective of the strain employed. Thus, taken together, previous and present results indicate that HA may exert some anticandidal effects, but exclusively at massive doses and only at early time points, likely as long as fungi are exponentially growing.

Concerning C. glabrata and C. parapsilosis, our results provide the first evidence on their susceptibility to HA. In particular, both strains are inhibited to a similar extent and in a dose-dependent manner. The only exception is HA1 that, unexpectedly, has little or no effects on C. glabrata, for reasons that remain to be established. Overall, we may conclude that HA provides an inhibitory signal also to fungal pathogens, although to a different extent, depending on the species considered and the dose of HA employed.

Overall, our findings show that different microbial species and even different strains belonging to the same species are differentially affected by a high molecular weight HA, in dose-dependent fashion. Several hypotheses may be forwarded to explain these findings. We cannot exclude the occurrence of non specific antimicrobial mechanisms. For example, the pronounced ability of this polysaccharide to absorb and retain water may account for the impaired and/or delayed growth of some of the tested microorganisms, after a suitable time-related adaptation (Figs. 3c, 4a). Also, the interaction between HA and specific microbial surface moieties, shared only among certain strains, may influence biological activities, such as bacterial co-aggregation; this hypothesis is in line with the recent observation that HA exerts distinct antiviral effects depending upon the virus [10]. Finally, as described above, unexpectedly no advantage is provided by HA to microorganisms commonly considered hyaluronidase producers, such as Gram positive bacteria. In this respect, studies performed in minimal medium supplemented with HA as the unique carbon source, indicate that none of the strains employed in the present study are able to grow (data not shown), suggesting that either hyaluronidases are not adequately produced or that the HA here assessed is not susceptible to microbial hydrolysis. Further wide-spectrum studies are warranted to investigate these hypotheses.

\section{Conclusions}

In conclusion, our study provides evidence on the effects of HA against a wide range of microbial agents, many of which are known opportunistic pathogens and/or cause of common iatrogenic infections. Our long-term kinetic study allows to distinguish among early vs transient vs continuous HA inhibitory effects on microbial agents; to our opinion, this is a critical aspect since exogenous HA persists for long time in tissues.

We can summarize our data by clustering the investigated microbial strains in three groups: (a) those that show a dose-dependent growth inhibition by HA, namely staphylococci, enterococci, S. mutans, two out of three E. coli strains, P. aeruginosa, C. glabrata and C. parapsilosis; (b) those that are not affected by HA, i.e. E. coli ATCC 13768 strain and C. albicans; (c) S. sanguinis that seems to be transiently favoured by $\mathrm{HA}$ at $4 \mathrm{mg} / \mathrm{ml}$. Whether and to what extent these findings may have an in vivo counterpart remains indeed an open question. Although with its intrinsic limitations, this in vitro approach has allowed wide spectrum analysis and long term assessment of HA biological activity on bacterial and fungal cells, as a preliminary and necessary step toward a better comprehension of the peculiarities of HA.

Acknowledgments The Authors are grateful to IBSA (Institut Biochimique SA, CH-6903 Lugano), that founded part of the research activity and the salary of M.C.

\section{References}

1. Romagnoli M, Belmontesi M. Hyaluronic acid-based fillers: theory and practice. Clin Dermatol. 2008;26:123-59.

2. Vazquez JR, Short B, Findlow AH, Nixon BP, Boulton AJ, Armstrong DG. Outcomes of hyaluronan therapy in diabetic foot wounds. Diabetes Res Clin Pract. 2003;59:123-7.

3. Slevin M, Krupinski J, Gaffney J, Matou S, West D, Delisser H, Savani RC, Kumar S. Hyaluronan-mediated angiogenesis in vascular disease: uncovering RHAMM and CD44 receptor signalling pathways. Matrix Biol. 2007;26:58-68.

4. Gao F, Yang CX, Mo W, Liu YW, He YQ. Hyaluronan oligosaccharides are potential stimulators to angiogenesis via RHAMM mediated signal pathway in wound healing. Clin Invest Med. 2008;31:106-16.

5. Campo GM, Avenoso A, Campo S, D'Ascola A, Nastasi G, Calatroni A. Molecular size hyaluronan differently modulates toll-like receptor-4 in LPS-induced inflammation in mouse chondrocytes. Biochimie. 2010;92:204-15.

6. Campo GM, Avenoso A, Campo S, D'Ascola A, Nastasi G, Calatroni A. Small hyaluronan oligosaccharides induce inflammation by engaging both toll-like-4 and CD44 receptors in human chondrocytes. Biochem Pharmacol. 2010;80:480-90.

7. Jiang D, Liang J, Noble PW. Hyaluronan as an immune regulator in human diseases. Physiol Rev. 2011;91:221-64.

8. Tiunnikov GI, Kostina GA, Radaeva IF, Bakulina LF. Effects of hyaluronic acid preparation on the development of herpetic infection in cell culture. Vopr Virusol. 2002;47:37-9.

9. Patterson RL, Peterson DA, Deinhardt F, Howard F. Rubella and rheumatoid arthritis: hyaluronic acid and susceptibility of cultured rheumatoid synovial cells to viruses. Proc Soc Exp Biol Med. 1975;149:594-8.

10. Cermelli C, Cuoghi A, Scuri M, Bettua C, Neglia RG, Ardizzoni A, Blasi E, Iannitti T, Palmieri B. In vitro evaluation of antiviral 
and virucidal activity of a high molecular weight hyaluronic acid. Virol J. 2011;8:141-8.

11. Costagliola C, Del Prete A, Winkler NR, Carpineto P, Ciancaglini M, Piccolomini R, Mastropasqua L. The ability of bacteria to use Na-hyaluronate as a nutrient. Acta Ophtalmol Scand. 1996;74: 566-8.

12. Zhang M, McDonald FM, Sturrock SS, Charnock SJ, HumpherySmith I, Black GW. Group A streptococcus cell-associated pathogenic proteins as revealed by growth in hyaluronic acidenriched media. Proteomics. 2007;7:1379-90.

13. Pirnazar P, Wolinsky L, Nachnani S, Haake S, Pilloni A, Bernard GW. Bacteriostatic effects of hyaluronic acid. J Periodontol. 1999;70:370-4.

14. Bardavio J, Miserachs N, Favà P, Ollé M, Rodriguez Y. Estudio del crecimiento bacteriano in vitro en sustancias viscoelasticas. Annals d'Oftalmologia. 2003;11:239-44.

15. Carlson GA, Dragoo JL, Samimi B, Bruckner DA, Bernard GW, Hedrick M, Benhaim P. Bacteriostatic properties of biomatrices against common orthopaedic pathogens. Biochem Biophys Res Commun. 2004;321:472-8.

16. Tang ZH, Hou CL, Chen QQ. Experimental study on bacteriostasis of chitosan and sodium hyaluronate. Zhongguo Xiu Fu Chong Jian Wai Ke Za Zhi. 2002;16:259-61.

17. Kang JH, Kim YY, Chang JY, Kho HS. Influences of hyaluronic acid on the anticandidal activities of lysozyme and the peroxidase system. Oral Dis. 2011 (in press).
18. Lauderdale TL, Chapin KC, Murray PR. Reagents. In: Murray PR, Baron EJ, Pfaller MA, Tenover FC, Yolken RH, editors. Manual of clinical microbiology, 7th edn. ASM Press, Washington D.C.; 1999. pp. 1665-73.

19. Radaeva IF, Kostina GA, Il'ina SG, Kostyleva RN. Antimicrobial activity of hyaluronic acid. Zh Mikrobiol Epidemiol Immunobiol. 2001;1:74-5.

20. UNI EN 13697. Chemical disinfectants and antisepticsQuantitative non-porous surface test for the evaluation of bactericidal and/or fungicidal activity of chemical disinfectants used in food, industrial, domestic and institutional areas-Test method and requirements without mechanical action (phase 2/step 2); 2001.

21. UNI EN 14349. Chemical disinfectants and antiseptics-Quantitative surface test for the evaluation of bacterial activity of chemical disinfectants and antiseptics used in veterinary field on non-porous surfaces without mechanical action-Test method and requirements (phase 2, step 2); 2005.

22. Wretlind B, Hedén L, Sjöberg L, Wadström T. Production of enzymes and toxins by hospital strains of Pseudomonas aeruginosa in relation to serotype and phage typing pattern. J Med Microbiol. 1973;6:91-100.

23. Brown MRW, Scott Foster JH, Clamp JR. Composition of Pseudomonas aeruginosa slime. J Biochem. 1969;112:521-5. 